\title{
Modeling and Model Predictive Power and Rate Control of Wireless Communication Networks
}

\author{
Cunwu Han, Dehui Sun, Song Bi, Lei Liu, and Zhengxi Li \\ Beijing Key Laboratory of Fieldbus Technology and Automation, North China University of Technology, Beijing 100144, China \\ Correspondence should be addressed to Cunwu Han; cwhan@ncut.edu.cn
}

Received 6 December 2013; Accepted 29 May 2014; Published 17 June 2014

Academic Editor: Aderemi Oluyinka Adewumi

Copyright (C) 2014 Cunwu Han et al. This is an open access article distributed under the Creative Commons Attribution License, which permits unrestricted use, distribution, and reproduction in any medium, provided the original work is properly cited.

A novel power and rate control system model for wireless communication networks is presented, which includes uncertainties, input constraints, and time-varying delays in both state and control input. A robust delay-dependent model predictive power and rate control method is proposed, and the state feedback control law is obtained by solving an optimization problem that is derived by using linear matrix inequality (LMI) techniques. Simulation results are given to illustrate the effectiveness of the proposed method.

\section{Introduction}

Power and rate control for wireless communication networks has attracted a great deal of interest during recent decades. The objective of power and rate control is to guarantee the quality of service (QoS) with the lowest transmit power and the maximum transmission data rate in order to maximize the capacity and throughput of the network. However, low power may lead to the degradation of communication quality or even unreliable connection, while high data rates may cause data bursts in the network. Therefore, transmit power and transmission rate must be effectively controlled for wireless communication networks.

There have been a lot of effective power and rate control methods reported in the literature. Generally speaking, the methods can be broadly classified into three categories: (i) resource allocation under perfect power control [1-4]; that is, the received power is well controlled at the target level, and then the transmission rate and power are optimised by maximizing the throughput, (ii) a snap-shot evaluation of the power and rate allocations pertaining to a given realisation of channel gains for each of the users $[5,6]$, and (iii) power control based on resource allocation [7-14]; that is, the optimal transmission rate can be obtained according to the resource allocation, and the optimal target signal-toinference ratio (SIR) can be relatively determined, and then the power control is used to determine an optimal transmit power for each user to drive its actual SIR towards the optimal target SIR. The third method will be used in this paper.

System modeling is an important requirement for power and rate control of wireless communication networks. A simplified linear system model was presented in [7], which considered round-trip delay and treated the effects of channel fading and interference as an additive disturbance. A frame/slot structure was presented in [8], which was used to implement practically a power and rate control system. A high order model as a cascade system was presented in $[9,10]$, which was used to cancel the effect of time delay. However, parameter uncertainties were not considered in these models. State space models with and without uncertain dynamics were presented in [11], but the time delay was not considered. An extended version of state space model in [11] with state delay was presented in [12], but it only considered the time delay in state and in rate control, while the time delay in input and in power control was not considered. In addition, all the models did not consider the time-varying delays and input constraints. Therefore, a new system model is needed for power and rate control to increase the QoS, which should include uncertainties, input constraints, and time-varying state and input delays in both rate control and power control. This motivates the work of this paper. 
A challenge issue in power and rate control is to maintain robustness to time delay $[9,10,12]$. To compensate for the effect of time delay, power and rate control algorithms have been presented by using a multiple-mode Smith prediction filter in [7] and by using a high order model in $[9,10]$. From a system-theoretic perspective, a power and rate control algorithm for wireless networks with state delay was presented in [12], which minimized the bound on the error variance between the desired and actual SIR. However, almost all existing power and rate control methods are limited to consider known and constant time delay. Unfortunately, the time delay is often unknown and even time-varying in real network environments.

In the previous work, we presented power and rate control algorithms for wireless networks with time-varying state delay via adaptive control technique [13] and via robust $H_{\infty}$ control approach [14]. However, as in [12], the time delay only in rate control was considered, while the time delay in power control was not considered. In fact, the power control is more sensitive to time delay than the rate control. Additionally, only the state time delay was considered in [12-14], but the input delay was not considered. To the best knowledge of the authors, research on power and rate control for wireless communication networks with time-varying delays in both state and control input has not been investigated, which is another motivation of this paper.

Another challenge issue in power and rate control is to maintain robustness to uncertainties and input constraints. It is well known that robust control with linear matrix inequality (LMI) is a powerful technique to cope with uncertainties, and model predictive control (MPC) has strong ability to handle input constraints $[15,16]$. Therefore, a robust MPC with LMI can be extended to power and rate control for wireless communication networks with uncertainties and input constraints, which is the third motivation of this paper.

The main contributions of this paper are as follows. (1) In the control theoretic framework, a novel power and rate control system model is presented, which includes uncertainties, input constraints, and time-varying state and input delays in both rate control and power control. (2) A robust model predictive power and rate control method is presented for wireless communication networks with uncertainties, input constraints, and time-varying state and input delays. The state feedback control law is obtained by solving an optimization problem that is derived by using LMI.

The rest of this paper is organized as follows. The power and rate control system model and problem formulation are presented in Section 2. A robust model predictive power and rate control algorithm is presented in Section 3. Simulation results are given in Section 4 . And Section 5 concludes the paper.

Notation. In this paper, $\mathbf{A}^{T}$ and $\mathbf{A}^{-1}$ denote the transpose and the inverse of a matrix $\mathbf{A}$, respectively; $R^{n \times m}$ denotes the set of all $n \times m$ real matrices; $\mathbf{A}>0(\mathbf{A}<0)$ means that $\mathbf{A}$ is symmetric positive definite (negative definite); I is an appropriately dimensioned identity matrix; $\operatorname{diag}\{\ldots\}$ denotes a block-diagonal matrix; $\|\mathbf{x}\|^{2}=\mathbf{x}^{T} \mathbf{x},\|\mathbf{x}\|_{\mathbf{P}}^{2}=\mathbf{x}^{T} \mathbf{P} \mathbf{x}$;

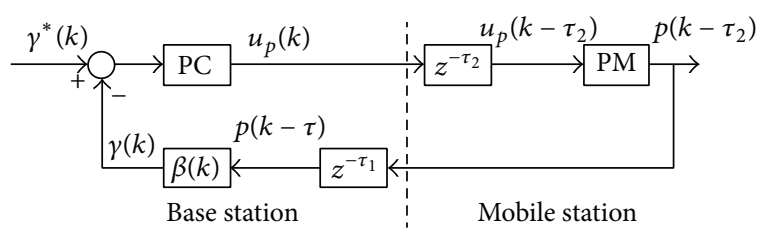

FIgURE 1: Time delays in power control.

the symmetric terms in a symmetric matrix are denoted by $*$; for example,

$$
\left[\begin{array}{ll}
\mathbf{X} & \mathbf{Y}^{T} \\
\mathbf{Y} & \mathbf{Z}
\end{array}\right]=\left[\begin{array}{ll}
\mathbf{X} & * \\
\mathbf{Y} & \mathbf{Z}
\end{array}\right]
$$

\section{System Model and Problem Formulation}

In this section, we briefly describe the time delay effects, power control, and rate control in Sections 2.1, 2.2, and 2.3, respectively, and then, in the control theoretic framework, derive the power and rate control system model for uncertain wireless communication networks with input constraints and time-varying delays in both state and control input in Section 2.4 and finally give the problem formulation in Section 2.5 .

2.1. Effects of Time Delays. In wireless communication networks, time delays are mainly due to the signal processing and signal transmission $[9,15]$. For convenience of analysis, as depicted in Figure 1 for the power control loop, let $\tau_{1}$ denote the time delay in uplink channel (including the signal processing delay at base station (BS) and the signal transmission delay from mobile station (MS) to BS), and let $\tau_{2}$ denote the time delay in downlink channel (including the signal processing delay at MS and the signal transmission delay from BS to $\mathrm{MS}$ ). The total round-trip delay in the network is $\tau=\tau_{1}+\tau_{2}$. Practical value of the round-trip delay is between 2 and 4 at the sampling rate of $800 \mathrm{~Hz}[10,17]$. To illustrate the effects of time delays in power control design, let us examine how a power control command $u_{p}(k+1)$ is produced. The effect of time delay in rate control design can be straightforward.

In the uplink power controller, with respect to the operation at MS, there are three main components related to the power control [18]: a quantization reconstructor, a power multiplier (PM), and a power limiter. At time instant $k$, the quantization reconstructor is employed to recover the power control update command $u_{p}(k)$ sent from BS, which is then fed into the power multiplier, resulting in the transmit power $p(k)$, which will be limited to the interval $\left[P_{\min }, P_{\max }\right]$ due to the physical limitation of power amplifier. Because of the downlink time delay $\tau_{2}$, the transmit power at MS should be $p\left(k-\tau_{2}\right)$. It is then transmitted to BS through the uplink channel with the uplink time delay $\tau_{1}$. That is, the transmitted power at MS is $p\left(k-\tau_{2}\right)$, and the received power at BS is $p\left(k-\tau_{2}-\tau_{1}\right)=p(k-\tau)$.

At BS, there are four major components related to the power control $[17,18]$ : an SIR measurement circuit, an SIR 
comparator, a power control circuit (PC), and a quantizer. The received SIR $\gamma(k)$, which is related to $p(k-\tau)$, is measured by the SIR measurement circuit. The tracking error can be obtained by comparison between the received SIR $\gamma(k)$ and the target SIR $\gamma^{*}(k)$ (which can be obtained by the rate controller as shown later). The error is fed into the power controller. The power controller generates a power control update command $u_{p}^{\prime}(k+1)$ according to an appropriate power control algorithm to make the received SIR $\gamma(k)$ track the target $\operatorname{SIR} \gamma^{*}(k)$ efficiently. The quantizer transfers the update signal from analog $u_{p}^{\prime}(k+1)$ to digital bits $u_{p}(k+1)$ based on a quantization scalar. $u_{p}(k+1)$ is then transmitted to MS through the downlink channel.

2.2. Power Control. According to the analysis in Section 2.1, the received SIR at BS for MS $i$ at time $k$ can be expressed as

$$
\gamma_{i}(k)=\frac{g_{i i}(k) p_{i}\left(k-\tau_{i}\right)}{\sum_{j=1, j \neq i}^{n} g_{i j}(k) p_{j}\left(k-\tau_{j}\right)+\sigma_{i}^{2}},
$$

where $\gamma_{i}(k)$ is a measurement of the actual SIR, $g_{i j}(k)$ represents the channel gain from the $j$ th MS to the intended BS of the $i$ th cell, $p_{j}(k)$ is the transmit power from the $j$ th MS, $n$ is the number of MSs using the same channel including the intracell and intercell users, $\sigma_{i}^{2}$ is the power of the white Gaussian noise at the receiver of BS, and $\tau_{j}$ is the round-trip delay. Now let

$$
\beta_{i}(k)=\frac{g_{i i}(k)}{\sum_{j=1, j \neq i}^{n} g_{i j}(k) p_{j}\left(k-\tau_{j}\right)+\sigma_{i}^{2}}
$$

denote the scaling factor that determines how $p_{i}\left(k-\tau_{i}\right)$ affects the achieved $\gamma_{i}(k)$ in $(2)$; that is,

$$
\gamma_{i}(k)=\beta_{i}(k) p_{i}\left(k-\tau_{i}\right) .
$$

By introducing logarithmic variables, with $\bar{\gamma}_{i}(k)=$ $\ln \left[\gamma_{i}(k)\right], \bar{\beta}_{i}(k)=\ln \beta_{i}(k)$, and $\bar{p}_{i}(k)=\ln p_{i}(k)$, we can rewrite (4) as, in $\mathrm{dB}$ scale,

$$
\bar{\gamma}_{i}(k)=\bar{\beta}_{i}(k)+\bar{p}_{i}\left(k-\tau_{i}\right),
$$

where $\bar{\beta}_{i}(k)$ is referred to as the effective channel gain.

Introduce random walk model for $\bar{\beta}_{i}(k)$ defined by [10]

$$
\bar{\beta}_{i}(k+1)=\bar{\beta}_{i}(k)+n_{i}(k),
$$

where $n_{i}(k)$ is a zero mean disturbance of variance $\sigma_{n}^{2}$ and is independent of $\bar{p}_{i}(k)$. This model is based on the assumption that MSs in the network do not jointly optimize their power levels in any centralized manner and only do so independently in a distributed sense.

The distributed power control (DPC) algorithm (in $\mathrm{dB}$ scale) for each MS is given by

$$
\bar{p}_{i}(k+1)=\bar{p}_{i}(k)+\alpha_{i}\left[\bar{\gamma}_{i}^{*}(k)-\bar{\gamma}_{i}(k)\right],
$$

where $\alpha_{i}$ is a step-size parameter that is allowed to vary from one MS to another, $\gamma_{i}^{*}(k)$ is a desired value of $\operatorname{SIR}$, and $\bar{\gamma}_{i}^{*}(k)=$ $\ln \gamma_{i}^{*}(k)$.
In order to make the actual SIR $\gamma_{i}(k)$ track the desired SIR $\gamma_{i}^{*}(k)$, as in [12], we employ a power control sequence $u_{i p}(k)$ in (7) as follows:

$$
\bar{p}_{i}(k+1)=\bar{p}_{i}(k)+\alpha_{i}\left[\bar{\gamma}_{i}^{*}(k)-\bar{\gamma}_{i}(k)\right]+b_{i p} u_{i p}(k),
$$

for a given factor $b_{i p}$ and control sequence $u_{i p}(k)$ to be determined.

Then from (5), (6), and (8), we find that the achieved $\bar{\gamma}_{i}(k)$ varies according to the following rule:

$$
\begin{aligned}
\bar{\gamma}_{i}(k+1) & \\
= & \bar{\beta}_{i}(k+1)+\bar{p}_{i}\left(k+1-\tau_{i}\right) \\
= & {\left[\bar{\beta}_{i}(k)+n_{i}(k)\right] } \\
& +\left\{\bar{p}_{i}\left(k-\tau_{i}\right)+\alpha_{i}\left[\bar{\gamma}_{i}^{*}\left(k-\tau_{i}\right)-\bar{\gamma}_{i}\left(k-\tau_{i}\right)\right]\right. \\
& \left.\quad+b_{i p} u\left(k-\tau_{i}\right)\right\} \\
= & {\left[\bar{\beta}_{i}(k)+n_{i}(k)\right]+\left[\bar{\gamma}_{i}(k)-\bar{\beta}_{i}(k)\right]+\alpha_{i} \bar{\gamma}_{i}^{*}\left(k-\tau_{i}\right) } \\
& -\alpha_{i} \bar{\gamma}_{i}\left(k-\tau_{i}\right)+b_{i p} u\left(k-\tau_{i}\right) \\
= & \bar{\gamma}_{i}(k)-\alpha_{i} \bar{\gamma}_{i}\left(k-\tau_{i}\right)+\alpha_{i} \bar{\gamma}_{i}^{*}\left(k-\tau_{i}\right) \\
& +b_{i p} u\left(k-\tau_{i}\right)+n_{i}(k) .
\end{aligned}
$$

Remark 1. Almost all existing power and rate control methods did not consider the time delay from the transmitted power to the received SIR, that is, assuming $\tau_{i}=0$ in (2). From the analysis of the dynamics as mentioned in Section 2.1, the effect of this time delay must be considered. This paper considers such a time delay which is mainly different from the existing power and rate control methods.

2.3. Rate Control. Let $f_{i}(k)$ denote the flow rate at MS $i$ at time $k$; for any MS in the network, the following flow-rate control algorithm is given by [12]

$$
\begin{aligned}
& f_{i}(k+1) \\
& \quad=f_{i}(k)+\mu\left[d(k)-c_{1}(k) f_{i}(k)-c_{2}(k) f_{i}\left(k-\tau_{i}\right)\right],
\end{aligned}
$$

where $\mu>0$ is a step-size parameter and $c_{1}(k)$ and $c_{2}(k)$ are measures of the amount of congestion in the network at time $k . d(k)$ controls the amount of rate increase per iteration, which is a zero mean random variable with variance $\sigma_{d}^{2} \cdot \tau_{i}$ is nonzero for any controller that incorporates round-trip delay time. Equation (10) is a typical rate control strategy incorporated in wireless communication networks and in computer networks.

According to Shannon's capacity formula, there is a plausible choice for SIR level in order to achieve a rate value $f_{i}(k)$; namely, the SIR level should be at least at a value $\gamma_{i}^{*}(k)$, that is, related to $f_{i}(k)$ given by

$$
f_{i}(k)=\frac{1}{2} \log _{2}\left[1+\gamma_{i}^{*}(k)\right] \text {. }
$$


Usually, during normal network operation, $\gamma_{i}^{*}(k) \gg 1$. Thus, $f_{i}(k)$ is proportional to $\log _{2} \gamma_{i}^{*}(k)$. Using the fact and (10), the desired SIR varies, in $\mathrm{dB}$ scale, according to the following rule:

$$
\begin{aligned}
\bar{\gamma}_{i}^{*}(k+1)= & {\left[1-\mu c_{1}(k)\right] \bar{\gamma}_{i}^{*}(k) } \\
& -\mu c_{2}(k) \bar{\gamma}_{i}^{*}\left(k-\tau_{i}\right)+\bar{\mu} d(k),
\end{aligned}
$$

where $\bar{\mu}=20 \mu / \log _{2}(10)$.

Like that mentioned in Section 2.2, as in [12], and considering the round trip time delay, we add a rate control sequence $u_{i f}(k)$ in (12) as follows:

$$
\begin{aligned}
\bar{\gamma}_{i}^{*}(k+1)= & {\left[1-\mu c_{1}(k)\right] \bar{\gamma}_{i}^{*}(k)-\mu c_{2}(k) \bar{\gamma}_{i}^{*}\left(k-\tau_{i}\right) } \\
& +b_{i f} u_{i f}\left(k-\tau_{i}\right)+\bar{\mu} d(k) .
\end{aligned}
$$

Remark 2. The power and rate control algorithms, presented in [10-12], just considered the time delay in rate control, while the time delay in power control was not considered, that is, in the case $\tau_{i}=0$ in (2). In fact, the power control is more sensitive to time delay than the rate control. So the time delay in power control must be considered. Furthermore, the time delay in control input was not considered in those algorithms, that is, in the case $\tau_{i}=0$ in $u_{i p}\left(k-\tau_{i}\right)$ in $(9)$ and in $u_{i f}\left(k-\tau_{i}\right)$ in (13).

2.4. Joint Power and Rate Control. For simplicity of notation, in the following, the MS index $i$ is dropped. And we introduce the two-dimensional state vector:

$$
\mathbf{x}(k)=\left[\begin{array}{c}
\bar{\gamma}(k) \\
\bar{\gamma}^{*}(k)
\end{array}\right] .
$$

Then from (9) and (13), we can obtain

$$
\begin{aligned}
\mathbf{x}(k+1)= & {\left[\begin{array}{cc}
1 & 0 \\
0 & 1-\mu c_{1}(k)
\end{array}\right] \mathbf{x}(k)+\left[\begin{array}{cc}
-\alpha & \alpha \\
0 & -\mu c_{2}(k)
\end{array}\right] \mathbf{x}(k-\tau) } \\
& +\left[\begin{array}{cc}
b_{p} & 0 \\
0 & b_{f}
\end{array}\right] \mathbf{u}(k-\tau)+\left[\begin{array}{c}
n(k) \\
\bar{\mu} d(k)
\end{array}\right]
\end{aligned}
$$

or, more compactly,

$$
\begin{aligned}
\mathbf{x}(k+1)= & \mathbf{A}(k) \mathbf{x}(k)+\mathbf{A}_{d}(k) \mathbf{x}(k-\tau) \\
& +\mathbf{B u}(k-\tau)+\boldsymbol{\omega}(k),
\end{aligned}
$$

where

$$
\begin{array}{cc}
\mathbf{A}(k)=\left[\begin{array}{cc}
1 & 0 \\
0 & 1-\mu c_{1}(k)
\end{array}\right], & \mathbf{A}_{d}(k)=\left[\begin{array}{cc}
-\alpha & \alpha \\
0 & -\mu c_{2}(k)
\end{array}\right], \\
\mathbf{B}=\left[\begin{array}{cc}
b_{p} & 0 \\
0 & b_{f}
\end{array}\right], & \boldsymbol{\omega}(k)=\left[\begin{array}{c}
n(k) \\
\bar{\mu} d(k)
\end{array}\right],
\end{array}
$$

where $\tau$ is a nonzero integer that incorporates round-trip time delay and $\boldsymbol{\omega}(k)$ is a zero mean random vector with covariance matrix:

$$
E\left\{\boldsymbol{\omega}(k) \boldsymbol{\omega}^{T}(k)\right\}=\left[\begin{array}{cc}
\sigma_{n}^{2} & 0 \\
0 & \bar{\mu}^{2} \sigma_{d}^{2}
\end{array}\right] .
$$

We now consider uncertainties that arise due to the lack of perfect knowledge about the network dynamics. For example, the congestion control parameters $c_{1}(k)$ and $c_{2}(k)$ are usually not known exactly and have to be estimated; the estimation process introduces errors in the assumed state-space model. The uncertainty in $c_{1}(k)$ can be modelled as [12]

$$
c_{1}(k)=c_{1}+D \mathbf{F}(k) \bar{d},
$$

where $\mathbf{F}(k)$ is a zero mean random noise with variance $\sigma_{F}^{2}, D$ and $\bar{d}$ are known scalars, and $c_{1}$ is unknown but bounded as

$$
c_{1, l} \leq c_{1} \leq c_{1, u}
$$

for some known positive scalars $\left\{c_{1, l}, c_{1, u}\right\}$. In other words, we allow for both deterministic and stochastic uncertainties in $c_{1}(k)$. In this way, the matrix $\mathbf{A}(k)$ in $(16)$ is not known exactly, but it can be modelled as $\mathbf{A}(k)=\mathbf{A}+\Delta \mathbf{A}(k)$, where

$$
\begin{gathered}
\mathbf{A}=\left[\begin{array}{cc}
1 & 0 \\
0 & 1-\mu c_{1}
\end{array}\right], \\
\Delta \mathbf{A}(k)=D \mathbf{F}(k) \mathbf{E}_{a},
\end{gathered}
$$

where

$$
\mathbf{E}_{a}=\left[\begin{array}{cc}
0 & 0 \\
0 & -\mu \bar{d}
\end{array}\right]
$$

Likewise, let $c_{2}$ be bounded as $c_{2, l} \leq c_{2} \leq c_{2, u}$. In this way, the matrix $\mathbf{A}_{d}(k)$ in (16) is also not known exactly but can be now modelled as $\mathbf{A}_{d}(k)=\mathbf{A}_{d}+\Delta \mathbf{A}_{d}(k)$, where

$$
\begin{gathered}
\mathbf{A}_{d}=\left[\begin{array}{cc}
-\alpha & \alpha \\
0 & -\mu c_{2}
\end{array}\right], \\
\Delta \mathbf{A}_{d}(k)=D \mathbf{F}(k) \mathbf{E}_{d},
\end{gathered}
$$

where

$$
\mathbf{E}_{d}=\left[\begin{array}{cc}
0 & 0 \\
0 & -\mu \bar{d}_{d}
\end{array}\right] .
$$

Then the power and rate control system with timevarying state and input delays and uncertainties can be written as

$$
\begin{gathered}
\mathbf{x}(k+1)=\mathbf{A}(k) \mathbf{x}(k)+\mathbf{A}_{d}(k) \mathbf{x}\left(k-\tau_{k}\right) \\
+\mathbf{B u}\left(k-\tau_{k}\right)+\boldsymbol{\omega}(k), \\
\mathbf{x}(k)=0, \quad k \in\left[-\tau_{M}, 0\right],
\end{gathered}
$$

where $\Delta \mathbf{A}(k)$ and $\Delta \mathbf{A}_{d}(k)$ are time-varying uncertain matrices of the form

$$
\left[\Delta \mathbf{A}(k), \Delta \mathbf{A}_{d}(k)\right]=D \mathbf{F}(k)\left[\mathbf{E}_{a}, \mathbf{E}_{d}\right],
$$


with $\mathbf{F}(k)^{T} \mathbf{F}(k) \leq \mathbf{I}$. Such parameter uncertainties $\Delta \mathbf{A}(k)$ and $\Delta \mathbf{A}_{d}(k)$ are said to be admissible. And it is assumed that, for the set of uncertainties $\boldsymbol{\Omega},\left[\mathbf{A}(k) \mid \mathbf{A}_{d}(k)\right] \in \mathbf{\Omega}$. $\tau_{k}$ is a roundtrip time-varying delay and satisfies

$$
0 \leq \tau_{m} \leq \tau_{k} \leq \tau_{M}
$$

where $\tau_{m}$ and $\tau_{M}$ are known lower and upper delay bounds. The control input is constrained by

$$
-\overline{\mathbf{u}} \leq \mathbf{u}(k+i) \leq \overline{\mathbf{u}}, \quad i \geq 0 .
$$

2.5. Problem Formulation. Define the following robust performance index at each time $k$ :

$$
\min _{\mathbf{u}(k+j \mid k), j \geq 0} \max _{\left[\mathbf{A}(k+j) \mid \mathbf{A}_{d}(k+j)\right] \in \mathbf{\Omega}} J(k),
$$

subject to

$$
\begin{gathered}
J(k)=\sum_{j=0}^{\infty}\left\{\|\mathbf{x}(k+j \mid k)\|_{\mathbf{Q}_{1}}^{2}+\|\mathbf{u}(k+j \mid k)\|_{\mathbf{R}}^{2}\right\}, \\
\mathbf{x}(k+j+1 \mid k) \\
=\mathbf{A}(k+j) \mathbf{x}(k+j \mid k)+\mathbf{A}_{d}(k+j) \\
\quad \times \mathbf{x}\left(k+j-\tau_{k} \mid k\right)+\mathbf{B u}(k+j \mid k)+\boldsymbol{\omega}(k+j), \\
-\overline{\mathbf{u}} \leq u(k+j)(=\mathbf{K} \mathbf{x}(k+j \mid k)) \leq \overline{\mathbf{u}}, \quad j \in[0, \infty),
\end{gathered}
$$

where $J(k)$ is the performance index function with respect to $\mathbf{x}(k), \mathbf{Q}_{1}>0$ and $\mathbf{R}>0$ are weighting matrices, $\phi(\mathbf{x}, \mathbf{u}, j)=$ $\|\mathbf{x}(k+j \mid k)\|_{\mathrm{Q}_{1}}^{2}+\|\mathbf{u}(k+j \mid k)\|_{R}^{2}$ is the stage cost, $\mathbf{x}(k+j \mid k)$ is the value of vector $\mathbf{x}$ at future time $k+j(j>0)$ predicted at time $k, x(k \mid k)=x(k)$, and $\mathbf{x}(k-i \mid k)=\mathbf{x}(k-i)$ for $i>1$.

Equations (29)-(30) are a constrained min-max optimization problem corresponding to a worst-case infinite-horizon MPC with a quadratic objective.

The goal of this paper is to find a state feedback control law $\mathbf{u}(k)=\mathbf{K} \mathbf{x}(k)$ for system (25) via the MPC strategy by solving the optimization problem (29)-(30).

Before ending this section, we introduce the following lemma, which is useful to prove our results.

Lemma 3 (see [19]). Let $\mathbf{D}, \mathbf{E}$, and $\mathbf{F}$ be real matrices of appropriate dimensions, with $\mathbf{F}^{\mathrm{T}} \mathbf{F} \leq \mathbf{I}$, and then there exists a scalar $\varepsilon \geq 0$, such that the following inequality holds:

$$
\mathbf{D F E}+\mathbf{E}^{\mathrm{T}} \mathbf{F}^{\mathrm{T}} \mathbf{D}^{T} \leq \varepsilon \mathbf{D D}^{T}+\varepsilon^{-1} \mathbf{E}^{\mathrm{T}} \mathbf{E}
$$

\section{Model Predictive Power and Rate Control}

In this section, we will design the robust state feedback controller for system (25) via the MPC strategy by solving the optimization problem (29)-(30). However, the exact solution to the min-max optimization problem (29)-(30) in general is not tractable. To obtain a practical optimization problem, following a similar approach as in [15], we will relax the min-max problem into another optimization problem which minimizes an upper bound on the worst value of the original cost function $J(k)$.

Consider a Lyapunov-Krasovskii functional at time $k$

$$
V(\mathbf{x}(k))=V_{1}(\mathbf{x}(k))+V_{2}(\mathbf{x}(k))+V_{3}(\mathbf{x}(k)),
$$

where

$$
\begin{aligned}
& V_{1}(\mathbf{x}(k))=\mathbf{x}^{T}(k) \mathbf{P} \mathbf{x}(k), \\
& V_{2}(\mathbf{x}(k))=\sum_{i=k-\tau_{k}}^{k-1} \mathbf{x}^{T}(i) \mathbf{P}_{\mathbf{d}} \mathbf{x}(i), \\
& V_{3}(\mathbf{x}(k))=\sum_{j=-\tau_{M}+1}^{-\tau_{m}} \sum_{i=k+j}^{k-1} \mathbf{x}^{T}(i) \mathbf{P}_{\mathbf{d}} \mathbf{x}(i),
\end{aligned}
$$

where $\mathbf{P}=\mathbf{P}^{T}>0, \mathbf{P}_{d}=\mathbf{P}_{d}^{T}>0$.

An upper bound on the worst value of the cost function $J(k)$ is obtained whenever the following inequality is satisfied for any $\left[\mathbf{A}(k+j \mid k) \mid \mathbf{A}_{d}(k+j \mid k)\right] \in \mathbf{\Omega}, j \geq 0$ :

$$
V(\mathbf{x}(k+j+1 \mid k))-V(\mathbf{x}(k+j \mid k)) \leq-\phi(\mathbf{x}, \mathbf{u}, k) .
$$

For $J(k)$ to be finite, we must have $\mathbf{x}(\infty \mid k)=0$, and hence $V(\mathbf{x}(\infty \mid k))=0$. Hence summing both sides of (36) from $j=0$ to $j=\infty$ yields $-V(\mathbf{x}(k \mid k)) \leq-J(k)$, and it follows that

$$
J(k) \leq V(\mathbf{x}(k \mid k)) \leq v(k)
$$

where $v(k)>0$ is defined to be the upper bound of the cost function $J(k)$. This implies

$$
\max _{\left[\mathbf{A}(k+j) \mid \mathbf{A}_{d}(k+j)\right] \in \mathbf{\Omega}, j \geq 0} J(k) \leq v(k) .
$$

Then, from (38), the original min-max optimization problem (29)-(30) is turned into the following optimization problem that minimizes this upper bound $v(k)$ :

$$
\min _{v(k), K, P, P_{d}} v(k)
$$

subject to (30) and (38).

Theorem 4. Consider the power and rate control system (25) subject to (26)-(28). If there exist matrices $\mathbf{Q}=\mathbf{Q}^{T}>0$, $\mathbf{Q}_{d}=\mathbf{Q}_{d}^{T}>0$, and $\mathbf{Y}, \mathbf{Z}>0$ with appropriate dimension, and scalars $\varepsilon>0$ and $v(k)>0$, such that the following optimization problem is solvable:

$$
\min _{v(k), Q, Q_{d}, Y, Z, \varepsilon} v(k),
$$

subject to 


$$
\left.\begin{array}{cccccccc}
-\mathbf{Q} & * & * & * & * & * & * & * \\
\left(\tau_{s}+1\right)^{1 / 2} \mathbf{Q} & -\mathbf{Q}_{d} & * & * & * & * & * & * \\
\mathbf{Q}_{1}^{1 / 2} \mathbf{Q} & \mathbf{0} & -v(k) \mathbf{I} & * & * & * & * & * \\
\mathbf{R}^{1 / 2} & \mathbf{0} & \mathbf{0} & -v(k) \mathbf{I} & * & * & * & * \\
\mathbf{0} & \mathbf{0} & \mathbf{0} & \mathbf{0} & -\mathbf{Q}_{d} & * & * & * \\
\mathbf{0} & \mathbf{0} & \mathbf{0} & \mathbf{0} & 0 & -v(k) \mathbf{I} & * & * \\
\mathbf{A} \mathbf{Q} & \mathbf{0} & \mathbf{0} & \mathbf{0} \quad \mathbf{A}_{d} \mathbf{Q}_{d}+\mathbf{B Y} & \mathbf{I} & \varepsilon \mathbf{D}^{T} \mathbf{D}-\mathbf{Q} & * \\
\mathbf{E}_{a} \mathbf{Q} & \mathbf{0} & \mathbf{0} & \mathbf{0} & \mathbf{E}_{d} \mathbf{Q}_{d} & \mathbf{0} & \mathbf{0} & -\varepsilon \mathbf{I}
\end{array}\right] \leq 0,
$$

where $\tau_{s}=\tau_{M}-\tau_{m}$, and

$$
\begin{gathered}
\mathbf{Q}=v \mathbf{P}^{-1}, \quad \mathbf{Q}_{d}=v \mathbf{P}_{d}^{-1}, \quad \mathbf{Y}=\mathbf{K} \mathbf{Q}_{d}, \\
\boldsymbol{\xi}_{3}(k)=\left[\mathbf{x}^{T}(k), \mathbf{x}^{T}(k-1), \ldots, \mathbf{x}^{T}\left(k-\tau_{m}\right),\right. \\
\left.\mathbf{x}^{T}\left(k-\tau_{m}-1\right), \ldots, \mathbf{x}^{T}\left(k-\tau_{M}\right)\right]^{T}, \\
\boldsymbol{\Lambda}=\operatorname{diag}\left(\mathbf{Q}, \frac{\mathbf{Q}_{d}}{\tau_{s}+1}, \ldots, \frac{\mathbf{Q}_{d}}{\tau_{s}+1}, \frac{\mathbf{Q}_{d}}{\tau_{s}}, \ldots, \mathbf{Q}_{d}\right),
\end{gathered}
$$

then the state feedback controller $\mathbf{u}(k)=\mathbf{K} \mathbf{x}(k)$ minimizes the upper bound of the cost function, where the feedback matrix $\mathbf{K}$ is obtained from the solution of (40)-(43) given by

$$
\mathbf{K}=\mathbf{Y} \mathbf{Q}_{d}^{-1} \text {. }
$$

The proof of Theorem 4 is presented in the Appendix.

Theorem 4 has given a sufficient condition for the existence of the robust MPC controller at time $k$. In order to prove the stability of the closed loop, a lemma is given as follows.

Lemma 5 (see [15]). If the optimization problem in Theorem 4 is feasible at time $k$, then it is feasible for all times $k+j, j>0$.

Next, we can give the following theorem to stabilize the closed-loop system.

Theorem 6. If the optimization problem (40)-(43) is feasible at time $k$, then the state feedback controller $\mathbf{u}(k)=\mathbf{K} \mathbf{x}(k)$ from Theorem 4 robustly asymptotically stabilizes the closedloop system.

Proof. It is known that the optimization problem (40)-(43) is feasible from Lemma 5. So we assume that $\mathbf{P}^{*}(k), \mathbf{P}_{d}^{*}(k)$ and $\mathbf{P}^{*}(k+1), \mathbf{P}_{d}^{*}(k+1)$ denote the optimal values of problem (40)-(43) at times $k$ and $k+1$, respectively, and $\mathbf{x}^{*}(k \mid k)$ and $\mathbf{x}^{*}(k+1 \mid k+1)$ are optimal state correspondingly.

Since $\mathbf{P}^{*}(k+1)$ and $\mathbf{P}_{d}^{*}(k+1)$ are optimal, whereas $\mathbf{P}^{*}(k)$, $\mathbf{P}_{d}^{*}(k)$ are feasible at time $k+1$, we have

$$
\begin{aligned}
& \mathbf{x}^{* T}(k+1 \mid k+1) \mathbf{P}^{*}(k+1) \mathbf{x}^{*}(k+1 \mid k+1) \\
& \leq \mathbf{x}^{* T}(k+1 \mid k+1) \mathbf{P}^{*}(k) \mathbf{x}^{*}(k+1 \mid k+1), \\
& \mathbf{x}^{T}(k+1-i \mid k+1) \mathbf{P}_{d}^{*}(k+1) \mathbf{x}(k+1-i \mid k+1) \\
& \quad \leq \mathbf{x}^{T}(k+1-i \mid k+1) \mathbf{P}_{d}^{*}(k) \mathbf{x}(k+1-i \mid k+1) .
\end{aligned}
$$

From Theorem 4, we have an invariant set $\Gamma$ for the predicted states of system (25),

$$
\begin{array}{r}
\Gamma=\left\{\mathbf{x}(k+i \mid k) \in R^{n} \mid \xi_{3}^{T}(k+i \mid k) \Lambda_{3} \xi_{3}(k+i \mid k) \leq v\right\} \\
=\left\{\mathbf{x}(k+i \mid k) \in R^{n} \mid \xi_{3}^{T}(k+i \mid k) \Lambda \xi_{3}(k+i \mid k) \leq 1\right\}, \\
i \geq 0
\end{array}
$$

and $\mathbf{u}(k+j \mid k)=\mathbf{K} \mathbf{x}(k+j \mid k), j \geq 0$. Then for any $[\mathbf{A}(k) \mid$ $\left.\mathbf{A}_{d}(k)\right] \in \mathbf{\Omega}$, we have

$$
\begin{aligned}
& \mathbf{x}^{T}(k+1 \mid k) \mathbf{P}(k) \mathbf{x}(k+1 \mid k) \\
& \quad<\mathbf{x}^{T}(k \mid k) \mathbf{P}(k) \mathbf{x}(k \mid k), \quad \mathbf{x}(k \mid k) \neq 0 .
\end{aligned}
$$


Noting $\mathbf{x}(k+1-i \mid k+1)<\mathbf{x}(k+1-i \mid k), i=1,2, \ldots, \tau_{k}$, that implies

$$
\begin{array}{r}
\mathbf{x}^{T}(k+1-i \mid k+1) \mathbf{P}_{d}^{*} \mathbf{x}(k+1-i \mid k+1) \\
\leq \mathbf{x}^{T}(k+1-i \mid k) \mathbf{P}_{d}^{*} \mathbf{x}(k+1-i \mid k), \\
i=1,2, \ldots, \tau_{k} .
\end{array}
$$

Since the measured state

$$
\begin{aligned}
\mathbf{x}(k+1 \mid k+1)= & \mathbf{x}(k+1) \\
= & \mathbf{A}(k) \mathbf{x}(k \mid k)+\left(\mathbf{A}_{d}(k)+\mathbf{B K}\right) \\
& \times \mathbf{x}\left(k-\tau_{k} \mid k\right)+\boldsymbol{\omega}(k)
\end{aligned}
$$

for some $\left[\mathbf{A}(k) \quad \mid \quad \mathbf{A}_{d}(k)\right] \in \mathbf{\Omega}$, it must also satisfy the inequality (48). Combining this with (46) and (49) and according to (32)-(35), we can obtain that

$$
V\left(\mathbf{x}^{*}(k+1 \mid k+1)\right) \leq V\left(\mathbf{x}^{*}(k \mid k)\right) .
$$

Therefore, $V(\mathbf{x}(k))$ is a monotonically nonincreasing and bounded Lyapunov function. Hence, the closed-loop system is robustly asymptotically stable. This completes the proof.

\section{Simulation Results}

To illustrate the performance of the proposed algorithm, we simulate a network using the model proposed in [12] for the channel gain from the $i$ th MS to its BS. In this model, the channel gain is given by

$$
G_{i i}(k)=S_{0} d_{i i}^{-\delta_{i}}(k) \cdot 10^{0.1 \zeta_{i}(k)},
$$

where $S_{0}$ is a function of the carrier frequency and $d_{i i}^{-\delta_{i}}(k)$ is the exponential path loss, which depends on the distance $d_{i i}(k)$ from the MS $i$ to its BS and the path-loss exponent, $\delta_{i} \in$ $R$, which typically takes values between 2 and 6 (we take 4 in the simulation). The term $10^{0.1 \zeta_{i}(k)}$ corresponds to the effect of shadowing (from building, terrain, or foliage), where $\zeta_{i}(k) \epsilon$ $R$ is a zero mean Gaussian random variable with variance $\sigma_{\zeta_{i}}^{2}$, which usually ranges between 6 and 12 . The values $c_{1}(k)$ and $c_{2}(k)$ are chosen as random variables between 0 and $0.5 . n(k)$ and $d(k)$ are zero mean with variance 0.01 . Moreover, take $\bar{\mu}=0.8, \alpha=0.2$.

As indicated in $[9,17]$, the practical value of the roundtrip delay is between 2 and 4 at the sampling rate of $800 \mathrm{~Hz}$ and is indicated in [18], and the typical value of the round-trip time delay (sum of the downlink and uplink delay) is between 0 and 4 for IS-95 wireless networks and is 2 for wideband code-division multiple access (WCDMA) wireless networks. In the simulations, we assume the time delay to be changed from 0 to 4 , that is, $0=\tau_{m} \leq \tau_{k} \leq \tau_{M}=4$.

The target SIR is set to be $10 \mathrm{~dB}$, and the input constraints are $0<\mathbf{u}(k+i) \leq 1, i \geq 0$. The parameters of MPC controller are set as follows: the prediction horizon is $N=5$ and the control horizon is $N_{u}=2$.
Figure 2 shows the tracking error between the actual SIR and the target SIR by using the proposed model predictive power and rate control method. It is confirmed that the proposed method has good tracking performance for the wireless communication network with time-varying delays, uncertainties, and input constraints. As comparison, the traditional robust power and rate control method presented in [12] is also used for the network with the same parameters; however, only the time delay in state and in rate control is considered, that is, in the case $\tau_{i}=0$ in (9) and in $u_{i f}\left(k-\tau_{i}\right)$ of (13), and the constant time delay $\tau=3$ is used in the design procedure. The tracking error is also shown in Figure 2. It is shown that the proposed method has less tracking error than the traditional robust power and rate control method.

\section{Conclusion}

Most existing power and rate control methods are limited to consider known and constant time delay, especially some methods only considering the time delay in state and in power control. This paper has presented a novel power and rate control system model, which includes uncertainties, input constraints, and time-varying delays in both state and input, not only in the rate control but also in the power control. A robust model predictive power and rate control method is proposed, and the state feedback control law is obtained by solving an optimization problem that is derived by using linear matrix inequality (LMI). Simulation result shows that the proposed method has better performance than the traditional power and rate control methods.

\section{Appendix}

Proof of Theorem 4. Under the state feedback control $\mathbf{u}(k)=$ $\mathbf{K} \mathbf{x}(k)$, the closed-loop system can be written as

$$
\mathbf{x}(k+1)=\overline{\mathbf{A}}(k) \mathbf{x}(k)+\overline{\mathbf{A}}_{d} \mathbf{x}\left(k-\tau_{k}\right)+\boldsymbol{\omega}(k),
$$

where

$$
\begin{gathered}
\overline{\mathbf{A}}(k)=\mathbf{A}+D \mathbf{F}(k) \mathbf{E}_{a}, \\
\overline{\mathbf{A}}_{d}(k)=\mathbf{A}_{d}+\mathbf{B K}+D \mathbf{F}(k) \mathbf{E}_{d} .
\end{gathered}
$$

Define $\Delta V(\mathbf{x}(k))=V(\mathbf{x}(k+1 \mid k))-V(\mathbf{x}(k \mid k))$; then along the solution of (25), we have

$$
\begin{aligned}
\Delta V(\mathbf{x}(k))= & \Delta V_{1}(\mathbf{x}(k))+\Delta V_{2}(\mathbf{x}(k))+\Delta V_{3}(\mathbf{x}(k)), \\
\Delta V_{1}(\mathbf{x}(k)) & =\mathbf{x}^{T}(k+1 \mid k) \mathbf{P} \mathbf{x}(k+1 \mid k)-\mathbf{x}^{T}(k) \mathbf{P} \mathbf{x}(k) \\
& =\boldsymbol{\xi}^{T}(k) \Pi_{1}^{T} \mathbf{P} \Pi_{1} \boldsymbol{\xi}(k)-\mathbf{x}^{T}(k) \mathbf{P} \mathbf{x}(k),
\end{aligned}
$$


where

$$
\begin{aligned}
& \boldsymbol{\xi}(k)=\left[\mathbf{x}^{T}(k), \mathbf{x}^{T}\left(k-\tau_{k}\right), \boldsymbol{\omega}(k)^{T}\right]^{T}, \quad \Pi_{1}=\left[\overline{\mathbf{A}}, \overline{\mathbf{A}}_{d}, \mathbf{I}\right], \\
& \Delta V_{2}(\mathbf{x}(k))=\sum_{i=k+1-\tau_{k+1}}^{k} \mathbf{x}^{T}(i) \mathbf{P}_{d} \mathbf{x}(i)-\sum_{i=k-\tau_{k}}^{k-1} \mathbf{x}^{T}(i) \mathbf{P}_{d} \mathbf{x}(i) \\
& =\mathbf{x}^{T}(k) \mathbf{P}_{d} \mathbf{x}(k)-\mathbf{x}^{T}\left(k-\tau_{k}\right) \mathbf{P}_{d} \mathbf{x}\left(k-\tau_{k}\right) \\
& +\sum_{i=k+1-\tau_{k+1}}^{k-1} \mathbf{x}^{T}(i) \mathbf{P}_{d} \mathbf{x}(i)-\sum_{i=k-\tau_{k}+1}^{k-1} \mathbf{x}^{T}(i) \mathbf{P}_{d} \mathbf{x}(i) \\
& \leq \mathbf{x}^{T}(k) \mathbf{P}_{d} \mathbf{x}(k)-\mathbf{x}^{T}\left(k-\tau_{k}\right) \mathbf{P}_{d} \mathbf{x}\left(k-\tau_{k}\right) \\
& +\sum_{i=k+1-\tau_{M}}^{k-1} \mathbf{x}^{T}(i) \mathbf{P}_{d} \mathbf{x}(i)-\sum_{i=k-\tau_{m}+1}^{k-1} \mathbf{x}^{T}(i) \mathbf{P}_{d} \mathbf{x}(i) \\
& =\mathbf{x}^{T}(k) \mathbf{P}_{d} \mathbf{x}(k)-\mathbf{x}^{T}\left(k-\tau_{k}\right) \mathbf{P}_{d} \mathbf{x}\left(k-\tau_{k}\right) \\
& +\sum_{i=k+1-\tau_{M}}^{k-\tau_{m}} \mathbf{x}^{T}(i) \mathbf{P}_{d} \mathbf{x}(i) \\
& \Delta V_{3}(\mathbf{x}(k)) \\
& =\sum_{j=-\tau_{M}+1}^{-\tau_{m}}\left[\sum_{i=k+j+1}^{k} \mathbf{x}^{T}(i) \mathbf{P}_{d} \mathbf{x}(i)-\sum_{i=k+j}^{k-1} \mathbf{x}^{T}(i) \mathbf{P}_{d} \mathbf{x}(i)\right] \\
& =\sum_{j=-\tau_{M}+1}^{-\tau_{m}}\left[\mathbf{x}^{T}(k) \mathbf{P}_{d} \mathbf{x}(k)-\mathbf{x}^{T}(k+j) \mathbf{P}_{d} \mathbf{x}(k+j)\right] \\
& =\tau_{s} \mathbf{x}^{T}(k) \mathbf{P}_{d} \mathbf{x}(k)-\sum_{i=k+1-\tau_{M}}^{k-\tau_{m}} \mathbf{x}^{T}(i) \mathbf{P}_{d} \mathbf{x}(i) .
\end{aligned}
$$

Combining (A.4) and (A.6), we have

$$
\Delta V(\mathbf{x}(k)) \leq \boldsymbol{\xi}^{T}(k)\left(\Theta_{1}+\Pi_{1}^{T} \mathbf{P} \Pi_{1}\right) \boldsymbol{\xi}(k),
$$

where $\boldsymbol{\Theta}_{1}=\operatorname{diag}\left(\left(\tau_{s}+1\right) \mathbf{P}_{d}-\mathbf{P},-\mathbf{P}_{d}, \mathbf{0}\right)$.

Considering (36) with $\mathbf{u}(k)=\mathbf{K x}(k)$, we obtain

$$
\Delta V(\mathbf{x}(k))+\phi(\mathbf{x}, \mathbf{u}, k)=\boldsymbol{\xi}^{T}(k)\left(\boldsymbol{\Theta}_{2}+\Pi_{1}^{T} \mathbf{P} \Pi_{1}\right) \boldsymbol{\xi}(k) \leq 0,
$$

where $\Theta_{2}=\operatorname{diag}\left(\left(\tau_{s}+1\right) \mathbf{P}_{d}-\mathbf{P}+\mathbf{Q}_{1}+\mathbf{K}^{\mathrm{T}} \mathbf{R K},-\mathbf{P}_{d}, \mathbf{0}\right)$. Then

$$
\Theta_{2}+\Pi_{1}^{T} \mathbf{P} \Pi_{1} \leq 0 .
$$
(41).

In the following, we will prove that (A.9) is equivalent to

Substituting $\mathbf{Q}=v(k) \mathbf{P}^{-1}$ and $\mathbf{Q}_{d}=v(k) \mathbf{P}_{d}^{-1}$ into (A.9), noting $\overline{\mathbf{A}}$ and $\overline{\mathbf{A}}_{d}$ in (A.1), and using Schur complement yield

$$
\boldsymbol{\Xi}+\boldsymbol{\Psi}_{D} \mathbf{F} \boldsymbol{\Psi}_{E}+\boldsymbol{\Psi}_{E}^{T} \mathbf{F}^{T} \boldsymbol{\Psi}_{D}^{T} \leq 0,
$$

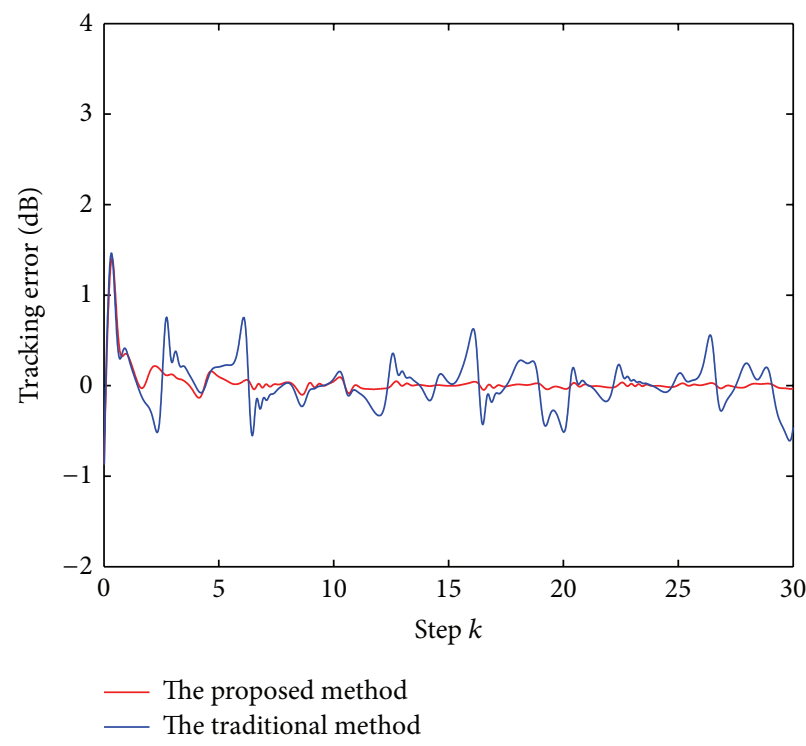

FIgURE 2: Tracking error between the actual SIR and the target SIR.

where

$$
\begin{gathered}
\boldsymbol{\Xi}=\left[\begin{array}{ccc}
\boldsymbol{\Phi}_{11} & * & * \\
\mathbf{0} & \boldsymbol{\Phi}_{22} & * \\
\overline{\mathbf{A}} & \overline{\mathbf{A}}_{d}+\mathbf{B K} & \mathbf{Q}
\end{array}\right] \leq 0, \\
\boldsymbol{\Phi}_{11}=\left(\tau_{s}+1\right) \mathbf{Q}_{d}^{-1}-\mathbf{Q}^{-1}+v^{-1}(k)\left(\mathbf{Q}_{1}+\mathbf{K}^{\mathrm{T}} \mathbf{R K}\right), \\
\boldsymbol{\Phi}_{22}=-\mathbf{Q}_{d}^{-1}, \quad \boldsymbol{\Psi}_{D}=\left[\begin{array}{lll}
\mathbf{0} & \mathbf{0} & \mathbf{D}^{T}
\end{array}\right]^{T}, \\
\boldsymbol{\Psi}_{E}=\left[\begin{array}{lll}
\mathbf{E}_{a} & \mathbf{E}_{d} & \mathbf{D}^{T}
\end{array}\right]^{T} .
\end{gathered}
$$

According to Lemma 3, there exists a scalar $\varepsilon>0$, such that the following inequality holds:

$$
\boldsymbol{\Psi}_{D} \mathbf{F} \boldsymbol{\Psi}_{E}+\boldsymbol{\Psi}_{E}^{T} \mathbf{F}^{\mathrm{T}} \boldsymbol{\Psi}_{D}^{T} \leq \varepsilon \boldsymbol{\Psi}_{\mathbf{D}} \boldsymbol{\Psi}_{D}^{T}+\varepsilon^{-1} \boldsymbol{\Psi}_{E}^{T} \boldsymbol{\Psi}_{E}
$$

Using Schur complement, it follows that

$$
\left[\begin{array}{cccc}
\Phi_{11} & * & * & * \\
\mathbf{0} & -\mathbf{Q}_{d}^{-1} & * & * \\
\overline{\mathbf{A}} & \overline{\mathbf{A}}_{d}+\mathbf{B K} & \varepsilon \mathbf{D}^{\mathrm{T}} \mathbf{D}-\mathbf{Q} & * \\
\mathbf{E}_{a} & \mathbf{E}_{d} & \mathbf{0} & -\varepsilon \mathbf{I}
\end{array}\right] \leq 0 .
$$

In order to design the controller and obtain the state feedback gain $\mathbf{K}$, pre- and postmultiplying (A.13) by $\operatorname{diag}\left(\mathbf{Q}, \mathbf{Q}_{d}, \mathbf{I}, \mathbf{I}\right)$, and considering $\mathbf{Y}=\mathbf{K} \mathbf{Q}_{d}$, we have

$$
\left[\begin{array}{cccc}
\overline{\mathbf{\Phi}}_{11} & * & * & * \\
\mathbf{0} & -\mathbf{Q}_{d} & * & * \\
\overline{\mathbf{A}} \mathbf{Q} & \overline{\mathbf{A}}_{d} \mathbf{Q}_{d}+\mathbf{B Y} & \varepsilon \mathbf{D}^{T} \mathbf{D}-\mathbf{Q} & * \\
\mathbf{E}_{a} \mathbf{Q} & \mathbf{E}_{d} \mathbf{Q}_{d} & \mathbf{0} & -\varepsilon \mathbf{I}
\end{array}\right] \leq 0
$$

where

$$
\overline{\mathbf{\Phi}}_{11}=\left(\tau_{s}+1\right) \mathbf{Q} \mathbf{Q}_{d}^{T} \mathbf{Q}-\mathbf{Q}+v^{-1}(k) \mathbf{Q} \mathbf{Q}_{1} \mathbf{Q}+v^{-1}(k) \mathbf{Y}^{T} \mathbf{R Y} .
$$


Then using Schur complement again, (41) is obtained. Furthermore, the feedback matrix $\mathbf{K}$ is given by $\mathbf{K}=\mathbf{Y Q}_{d}^{-1}$.

The next step of the proof involves the LMI (42). Equations (34) and (35) can be rewritten as

$$
\begin{aligned}
V_{2}(\mathbf{x}(k)) & =\sum_{i=k-\tau_{k}}^{k-1} \mathbf{x}^{T}(i) \mathbf{P}_{d} \mathbf{x}(i) \\
& \leq \sum_{i=k-\tau_{M}}^{k-1} \mathbf{x}^{T}(i) \mathbf{P}_{d} \mathbf{x}(i) \\
& =\xi_{1}^{T}(k-1) \Lambda_{1} \xi_{1}(k-1),
\end{aligned}
$$

where

$$
\begin{aligned}
\boldsymbol{\xi}_{1}(k-1)= & {\left[\mathbf{x}^{T}(k-1), \ldots, \mathbf{x}^{T}\left(k-\tau_{M}\right)\right]^{T}, } \\
\boldsymbol{\Lambda}_{1} & =\operatorname{diag}\left(\mathbf{P}_{d}, \ldots, \mathbf{P}_{d}\right), \\
V_{3}(\mathbf{x}(k)) & =\sum_{j=-\tau_{M}+1}^{-\tau_{m}} \sum_{i=k+j}^{k-1} \mathbf{x}^{T}(i) \mathbf{P}_{d} \mathbf{x}(i) \\
& =\boldsymbol{\xi}_{2}^{T}(k-1) \boldsymbol{\Lambda}_{2} \boldsymbol{\xi}_{2}(k-1),
\end{aligned}
$$

where

$$
\begin{gathered}
\boldsymbol{\xi}_{2}(k-1)=\left[\mathbf{x}^{T}(k-1), \mathbf{x}^{T}(k-2), \ldots, \mathbf{x}^{T}\left(k-\tau_{m}\right),\right. \\
\left.\mathbf{x}^{T}\left(k-\tau_{m}-1\right), \ldots, \mathbf{x}^{T}\left(k-\tau_{M}+1\right)\right]^{T}, \\
\boldsymbol{\Lambda}_{2}=\operatorname{diag}\left(\tau_{s} \mathbf{P}_{d}, \ldots, \tau_{s} \mathbf{P}_{d},\left(\tau_{s}-1\right) \mathbf{P}_{d}, \ldots, \mathbf{P}_{d}\right) .
\end{gathered}
$$

Summing (33), (A.16), and (A.18), we can conclude that

$$
V(\mathbf{x}(k))=\xi_{3}^{T}(k) \Lambda_{3} \xi_{3}(k),
$$

where

$$
\begin{gathered}
\boldsymbol{\xi}_{3}(k)=\left[\mathbf{x}^{T}(k), \mathbf{x}^{T}(k-1), \ldots, \mathbf{x}^{T}\left(k-\tau_{m}\right),\right. \\
\left.\mathbf{x}^{T}\left(k-\tau_{m}-1\right), \ldots, \mathbf{x}^{T}\left(k-\tau_{M}\right)\right]^{T}, \\
\Lambda_{3}=\operatorname{diag}\left(\mathbf{P},\left(\tau_{s}+1\right) \mathbf{P}_{d}, \ldots,\left(\tau_{s}+1\right) \mathbf{P}_{d}, \tau_{s} \mathbf{P}_{d}, \ldots, \mathbf{P}_{d}\right) .
\end{gathered}
$$

Combining (36) with (A.20), noting $\mathbf{Q}=v(k) \mathbf{P}^{-1}$ and $\mathbf{Q}_{d}=v(k) \mathbf{P}_{d}^{-1}$, and then using Schur complement, the LMIs (42) can be obtained.

Following [15], the input constraint (28) is equivalent to (43). This completes the proof.

\section{Conflict of Interests}

The authors declare that there is no conflict of interests regarding the publication of this paper.

\section{Acknowledgments}

This work is supported by the National Natural Science Foundation of China (61174116), the Beijing Natural Science Foundation (4142014), and the Scientific Research Foundation of North China University of Technology (20130017). The authors would like to thank the reviewers for their helpful suggestions and comments.

\section{References}

[1] M. Chiang, "Balancing transport and physical layers in wireless multihop networks: jointly optimal congestion control and power control," IEEE Journal on Selected Areas in Communications, vol. 23, no. 1, pp. 104-116, 2005.

[2] A. T. Hoang, Y.-C. Liang, and M. H. Islam, "Power control and channel allocation in cognitive radio networks with primary users' cooperation," IEEE Transactions on Mobile Computing, vol. 9, no. 3, pp. 348-360, 2010.

[3] N. H. Tran and C. S. Hong, "Joint rate and power control in wireless network: a novel successive approximations method," IEEE Communications Letters, vol. 14, no. 9, pp. 872-874, 2010.

[4] M. R. Musku, A. T. Chronopoulos, D. C. Popescu, and A. Ştefănescu, "A game-theoretic approach to joint rate and power control for uplink CDMA communications," IEEE Transactions on Communications, vol. 58, no. 3, pp. 923-932, 2010.

[5] F. Berggren and S.-L. Kim, "Energy-efficient control of rate and power in DS-CDMA systems," IEEE Transactions on Wireless Communications, vol. 3, no. 3, pp. 725-733, 2004.

[6] C. C. Chai, T. T. Tjhung, and L. C. Leck, "Combined power and rate adaptation for wireless cellular systems," IEEE Transactions on Wireless Communications, vol. 4, no. 1, pp. 6-13, 2005.

[7] B.-S. Chen, C.-Y. Yang, and S.-Y. Li, "Adaptive two-loop power tracking control in CDMA systems with the utility optimization," IEEE Transactions on Wireless Communications, vol. 7, no. 4, pp. 1358-1368, 2008.

[8] L. Zhao and J. W. Mark, "Joint rate and power adaptation for radio resource management in uplink wideband code division multiple access systems," IET Communications, vol. 2, no. 4, pp. 562-572, 2008.

[9] A. Möller, U. T. Jönsson, M. Blomgren, and F. Gunnarsson, "Congestion-based rate and power control in wireless cellular networks," in Proceedings of the IEEE Global Communications Conference (Globecom '11), Houston, Tex, USA, December 2011.

[10] A. Moller, U. T. Jönsson, M. Blomgren, and F. Gunnarsson, "Stability of rate and power control algorithms in wireless cellular networks," in Proceedings of the Joint 50th IEEE Conference on Decision and Control and European Control Conference (CDCECC '11), pp. 4535-4541, Orlando, Fla, USA, December 2011.

[11] A. Subramanian and A. H. Sayed, "Joint rate and power control algorithms for wireless networks," IEEE Transactions on Signal Processing, vol. 53, no. 11, pp. 4204-4214, 2005.

[12] A. Subramanian and A. H. Sayed, "A robust power and rate control method for state-delayed wireless networks," Automatica, vol. 41, no. 11, pp. 1917-1924, 2005.

[13] C. Han, D. Sun, Y. Shi, and S. Bi, "Adaptive power and rate control for wireless networks with time-varying delay," in Proceedings of the IASTED International Conference on Control and Applications (CA '13), pp. 60-66, Honolulu, Hawaii, USA, August 2013. 
[14] C. Han, D. Sun, and L. Liu, "Performance analysis of power and rate control for wireless networks with time-varying delay," in Proceedings of the IEEE International Conference on Computer Science and Automation Engineering (CSAE '13), pp. 1309-1313, Guangzhou, China, November 2013.

[15] M. V. Kothare, V. Balakrishnan, and M. Morari, "Robust constrained model predictive control using linear matrix inequalities," Automatica, vol. 32, no. 10, pp. 1361-1379, 1996.

[16] D. Q. Mayne, J. B. Rawlings, C. V. Rao, and P. O. M. Scokaert, "Constrained model predictive control: stability and optimality," Automatica, vol. 36, no. 6, pp. 789-814, 2000.

[17] H. Zhang and P. N. Pathirana, "Uplink power control via adaptive hidden-markov-model-based pathloss estimation," IEEE Transactions on Mobile Computing, vol. 12, no. 4, pp. 657-665, 2013.

[18] C.-Y. Yang and B.-S. Chen, "Robust power control of CDMA cellular radio systems with time-varying delays," Signal Processing, vol. 90, no. 1, pp. 363-372, 2010.

[19] L. Xie, "Output feedback Ho control of systems with parameter uncertainty," International Journal of Control, vol. 63, no. 4, pp. 741-750, 1996. 


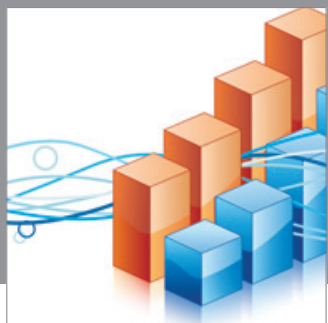

Advances in

Operations Research

mansans

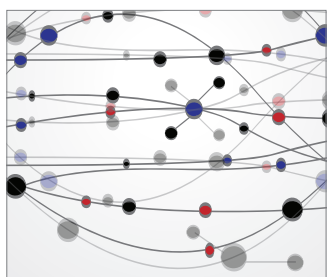

The Scientific World Journal
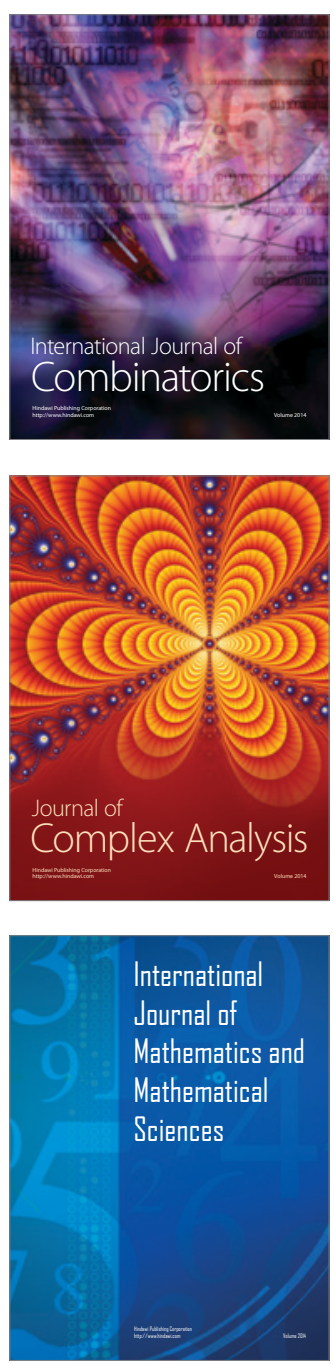
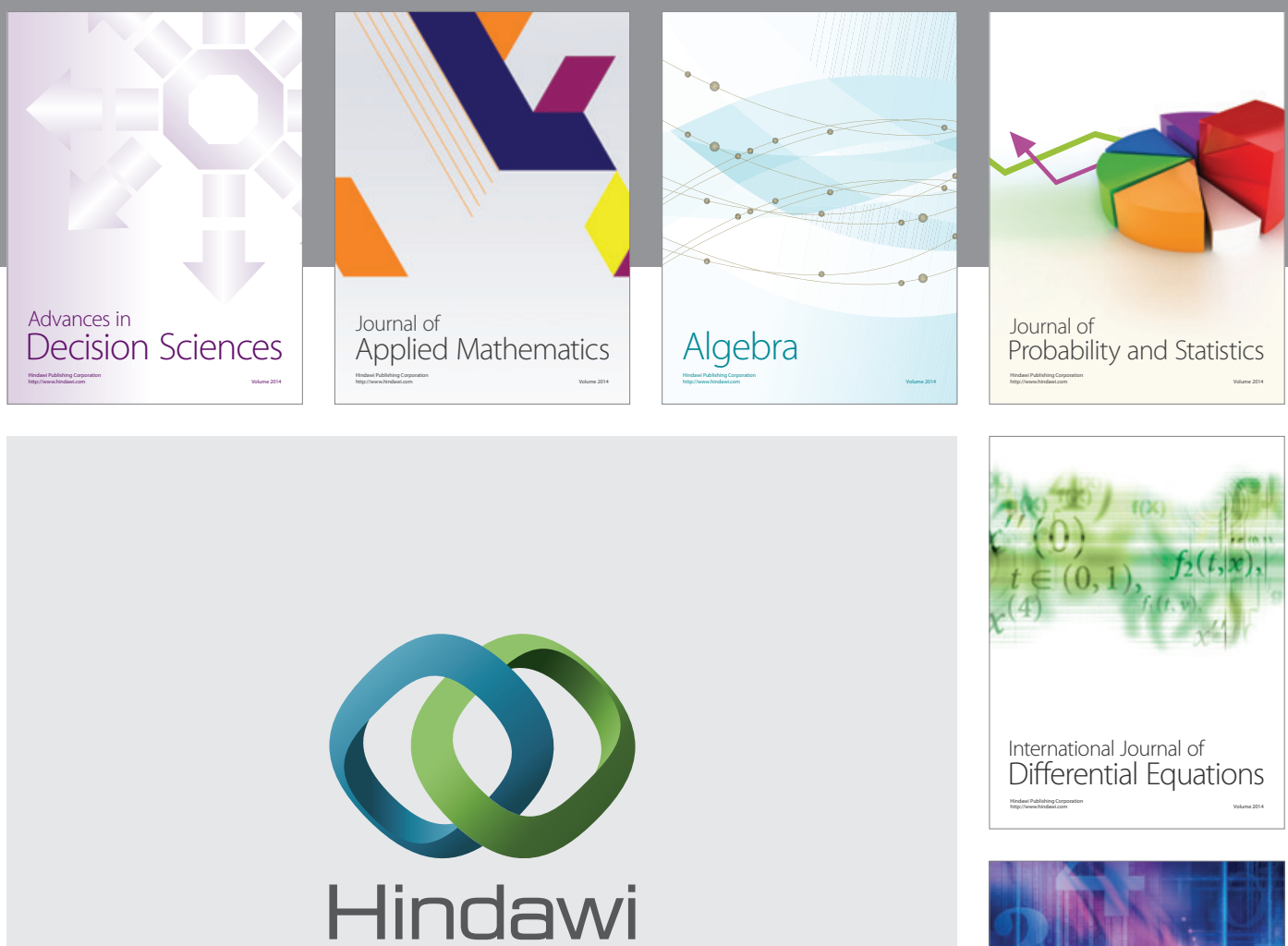

Submit your manuscripts at http://www.hindawi.com
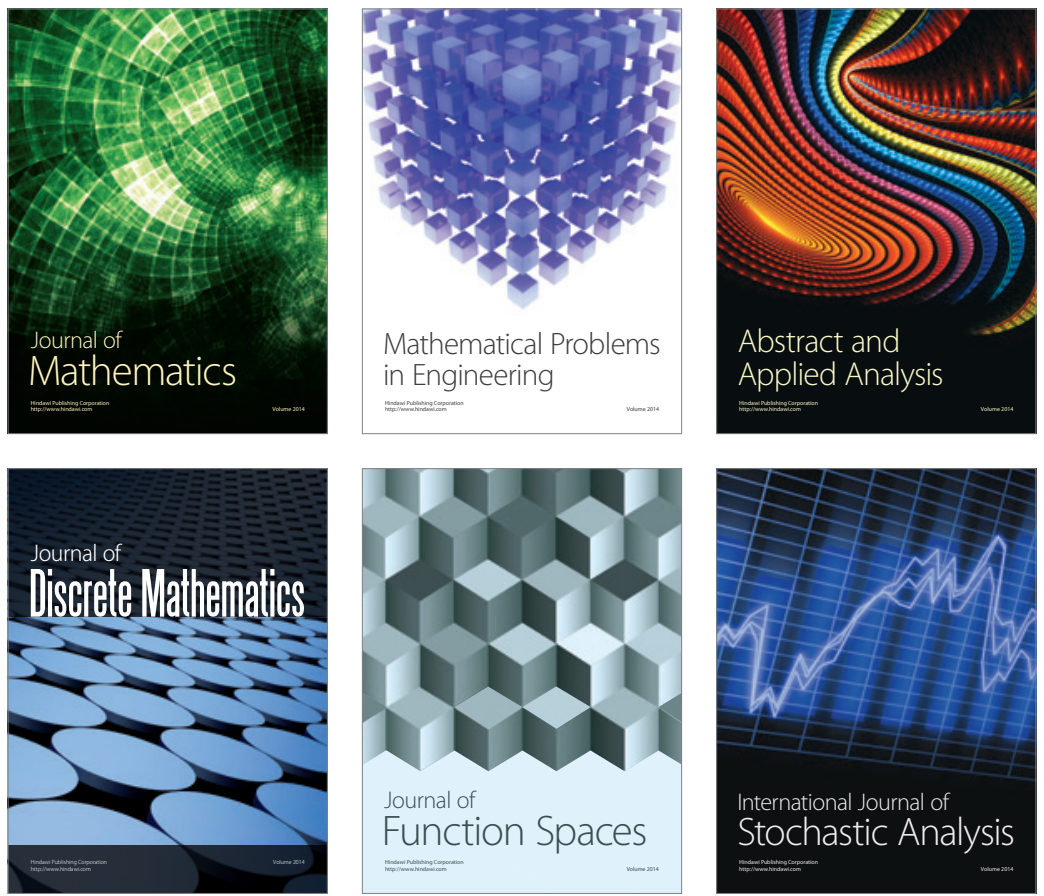

Journal of

Function Spaces

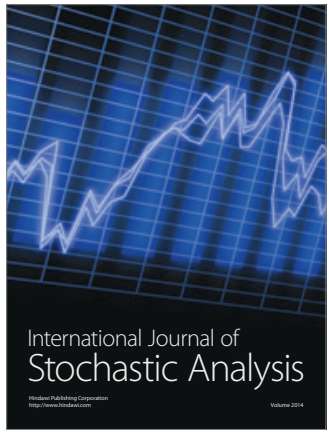

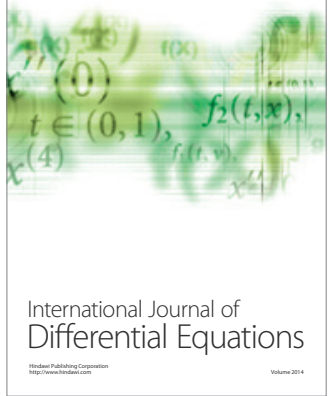
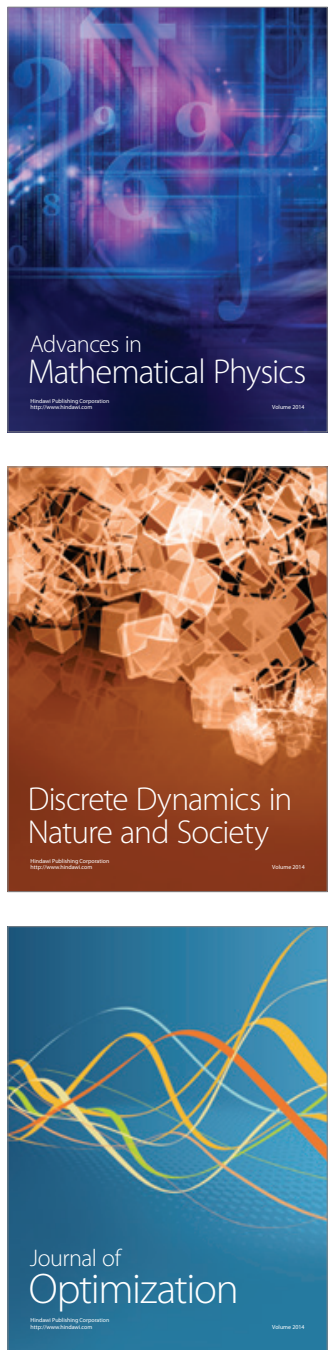